\section{Estudio histopatológico de tejido cardiaco de roedores infectados con Trypanosoma cruzi capturados en barrios suburbanos de Mérida, México}

\author{
Fernando Ucan-Euan ${ }^{1}$, Silvia Hernández-Betancourt ${ }^{1}$, Madeleine Arjona-Torres²,
} Alonso Panti-May ${ }^{3}$, Marco Torres-Castro ${ }^{4}$

\author{
${ }^{1}$ Bioecología Animal, Campus de Ciencias Biológicas y Agropecuarias, Universidad Autónoma de \\ Yucatán, Mérida, México \\ ${ }^{2}$ Laboratorio de Patología, Campus de Ciencias Biológicas y Agropecuarias, Universidad \\ Autónoma de Yucatán, Mérida, México \\ ${ }^{3}$ Doctorado en Ciencias Agropecuarias, Campus de Ciencias Biológicas y Agropecuarias, \\ Universidad Autónoma de Yucatán, Mérida, México \\ ${ }^{4}$ Laboratorio de Enfermedades Emergentes y Reemergentes, Centro de Investigaciones \\ Regionales "Dr. Hideyo Noguchi”, Universidad Autónoma de Yucatán, Mérida, México
}

Introducción. Trypanosoma cruzi es el agente causal de la tripanosomiasis americana, enfermedad endémica en México. Los roedores Mus musculus y Rattus rattus son reservorios del parásito, el cual invade las fibras cardiacas y desarrolla nidos parasitarios produciendo diversas lesiones. Los estudios histopatológicos en roedores naturalmente infectados son escasos. Objetivo. Describir los tipos y las frecuencias de las lesiones microscópicas en muestras de tejido cardiaco de $M$. musculus y $R$. rattus infectados con $T$. cruzi capturados en Mérida, México. Materiales y métodos. Los roedores se capturaron en los barrios suburbanos de Mérida. Se extrajo el tejido cardiaco y se procesó por la técnica de inclusión en parafina y tinción con hematoxilina y eosina. Su examen se hizo con un microscópico convencional y se determinaron todas las lesiones y su grado de afección.

Recibido: 29/11/17 Aceptado: $01 / 11 / 18$

Citación:

Ucan-Euan F, Hernández-Betancourt S, ArjonaTorres M, Panti-May A, Torres-Castro M. Estudio histopatológico de tejido cardiaco de roedores infectados con Trypanosoma cruzi capturados en barrios suburbanos de Mérida, México. Biomédica. 2019;39(Supl.2):32-43

https://doi.org/10.7705/biomedica.v39i3.4192

\section{Correspondencia:}

Marco Torres-Castro, Laboratorio de Enfermedades Emergentes y Reemergentes, Centro de Investigaciones Regionales "Dr. Hideyo Noguchi", Universidad Autónoma de Yucatán, Avenida Itzáes $N^{\circ}$. 490, Col. Centro, CP. 97000, Mérida, México Teléfonos: (+52) (999) 924 5809, 924 5755; fax: (999) 9236120

antonio.torres@correo.uady.mx

Contribución de los autores:

Fernando Ucan-Euan: preparación de las muestras, análisis histopatológico e interpretación de los resultados

Silvia Hernández-Betancourt: diseño del muestreo y del estudio y revisión del manuscrito final Madeleine Arjona-Torres: análisis histopatológico, interpretación de resultados y revisión del manuscrito final

Alonso Panti-May: trabajo de campo y revisión del manuscrito final

Marco Torres-Castro: trabajo de campo, escritura y revisión del manuscrito final

Financiación:

Este estudio fue parcialmente apoyado por el proyecto FOMIX-CONACYT-YUC-2008-108929 ("Sensibilidad y vulnerabilidad de los ecosistemas costeros del sureste de México ante el cambio climático global").

Conflicto de intereses:

Los autores declaran que no existe ningún conflicto de intereses para la publicación del presente manuscrito.
Resultados. Se trabajaron ocho muestras de tejido de $M$. musculus y siete de $R$. rattus. Se encontraron nidos parasitarios en siete del total de las muestras: en 3 de las 8 de $M$. musculus y en 4 de las 7 de $R$. rattus. Se observaron infiltrados inflamatorios en todas las muestras. Otras lesiones fueron la degeneración de las fibras cardiacas (8/15), la congestión de los vasos sanguíneos (6/15) y la necrosis (5/15).

Discusión. Las lesiones observadas están descritas en los modelos animales experimentales y en los humanos con tripanosomiasis americana. Los infiltrados inflamatorios se han descrito como la lesión más significativa en los humanos y en los reservorios en la etapa crónica de la enfermedad.

Conclusión. Las lesiones observadas están asociadas con la infección con T. cruzi, lo cual confirma que los roedores estudiados son reservorios de este parásito.

Palabras clave: Trypanosoma cruzi, ratones, ratas.

\section{Histopathological study in cardiac tissue of rodents infected with Trypanosoma} cruzi, captured in suburbs of Mérida, México

Introduction: Trypanosoma cruzi is the causal agent of the American trypanosomiasis, an endemic disease in México. The commensal rodents Mus musculus and Rattus rattus are reservoirs of this parasite, which invades cardiac fibers and develops parasite nests causing various lesions. Histopathological studies in naturally infected rodents are scarce. Objective: To describe the types and frequencies of microscopic lesions in cardiac tissue of $M$. musculus and $R$. rattus infected with $T$. cruzi captured in Mérida, México.

Materials and methods: The rodents were captured in suburban environments of Mérida. Cardiac tissue was extracted and processed by the paraffin inclusion technique and hematoxylin and eosin stained. The observation was made with a conventional microscope and all the lesions, as well as their degree, were identified.

Results: Eight tissue samples of $M$. musculus and seven of $R$. rattus were studied. Parasite nests were found in $7 / 15$, specifically $3 / 8$ in $M$. musculus and $4 / 7$ in $R$. rattus. The inflammatory infiltrate was the most frequent lesion. Other lesions were: Degeneration of cardiac fibers (8/15), congestion of blood vessels (6/15), and necrosis (5/15).

Discussion: The lesions we observed have been described in experimental animal models and in humans with American trypanosomiasis. The inflammatory infiltrate has been identified as the most significant lesion in humans and reservoirs in the chronic stage of the disease. Conclusion: The lesions we described are associated with $T$. cruzi infection, which confirms that the rodents studied are reservoirs of this parasite.

Keywords: Trypanosoma cruzi, mice, rats. 
Trypanosoma cruzi es un protozoario parásito intracelular obligado, causante de la tripanosomiasis americana o enfermedad de Chagas, padecimiento endémico en distintos países de Latinoamérica (1). En el sureste mexicano -incluido el Estado de Yucatán-, T. cruzi se transmite principalmente por las heces y la picadura del insecto vector Triatoma dimidiata; sin embargo, otros triatominos, como Panstrongylus rufotuberculatus, Eratyrus cuspidatus, Triatoma nitida y Triatoma hegneri, pueden participar en el ciclo de transmisión (2).

El contagio en humanos también puede ocurrir por la exposición a sangre contaminada durante el periodo perinatal, por transfusiones sanguíneas, en accidentes de laboratorio o por ingestión de alimentos contaminados (3). Una vez infectado el huésped o reservorio, $T$. cruzi afecta gran variedad de células y tejidos, incluidos macrófagos, músculo liso, músculo estriado, músculo cardiaco, fibroblastos y células de Schwann (4).

Las manifestaciones de la tripanosomiasis americana incluyen desde casos asintomáticos hasta cuadros clínicos crónicos atípicos, lo cual resulta en la fluctuación de las tasas de mortalidad (5). La fase parasitémica aguda se caracteriza por fiebre de $38^{\circ} \mathrm{C}$ y, después de dos a tres semanas de infección, puede presentarse edema facial, de piernas y pies, así como hepatoesplenomegalia y linfadenopatía axilar e inguinal moderadas (6). El periodo crónico se manifiesta al cabo de diez años o más de la primoinfección y se desarrolla en el $30 \%$ de los pacientes con signos cardiacos graves como cardiomegalia y alteraciones histológicas como necrosis celular, infiltrados inflamatorios intersticiales (focales o difusos), edema y fibrosis intersticial (7). Asimismo, T. cruzi coloniza ampliamente las fibras cardiacas para desarrollarse y multiplicarse en los denominados nidos con formas de amastigotes, dando origen al 'corazón chagásico' $(8,9)$.

La infección natural con T. cruzi en roedores sinantrópicos (comensales) o silvestres se ha descrito en diversos estudios serológicos y moleculares (10-13) mediante los cuales se ha establecido que Mus musculus y Rattus rattus son algunos de los principales reservorios del parásito y participan en los ciclos de transmisión domésticos, peridomésticos y silvestres (12-14). Específicamente en Yucatán, México, la infección natural con $T$. cruzi se ha identificado en $M$. musculus (ratón común) y $R$. rattus (rata negra), lo que ha puesto en evidencia su probable participación en el ciclo de transmisión en la región $(15,16)$. Estos hallazgos resultan relevantes debido a la cercanía y la amplia presencia de estos animales en asentamientos urbanos del Estado (17).

El diagnóstico de la enfermedad de Chagas puede hacerse mediante pruebas serológicas y moleculares dependiendo del mecanismo de transmisión, la etapa evolutiva del padecimiento y la sensibilidad y especificidad de las pruebas (18). No obstante, el estudio histopatológico de los tejidos u órganos infectados (principalmente el músculo cardiaco) representan una alternativa complementaria para el diagnóstico y también ayudan en la interpretación de la fisiopatología de la enfermedad en los casos fatales $(9,19)$.

Los hallazgos histopatológicos de los diversos estudios a nivel mundial se han descrito solo brevemente y se registran poco en roedores sinantrópicos y en otros reservorios o huéspedes naturalmente infectados con $T$. cruzi $(20,21)$. En este contexto, el objetivo del presente estudio fue describir los tipos y la frecuencia de las lesiones histológicas presentes en el tejido cardiaco de $M$. musculus y $R$. rattus naturalmente infectados con $T$. cruzi, capturados en los barrios suburbanos de Mérida, Yucatán, México. 


\section{Materiales y métodos}

\section{Sitio de estudio}

El presente estudio se llevó a cabo en los barrios Plan de Ayala Sur II

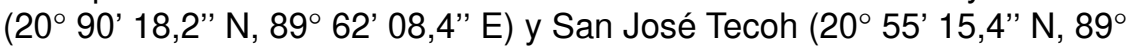
$37^{\prime} 35,6^{\prime \prime}$ E), ubicados al sur de la ciudad de Mérida, la cual es el mayor centro urbano del sureste del país. Ambos barrios están separados por una distancia menor de $3 \mathrm{~km}$. El clima predominante en los dos sitios es cálido subhúmedo con lluvias en verano (AW' 0). La temperatura media anual es de $26^{\circ} \mathrm{C}\left(36^{\circ} \mathrm{C}\right.$, máxima y $18^{\circ} \mathrm{C}$, mínima), con dos estaciones climáticas bien marcadas: de lluvia (mayo a octubre) y seca (noviembre a abril) (22).

El barrio Plan de Ayala Sur II se encuentra fuera de la periferia de Mérida, dentro de la Reserva Ecológica Cuxtal. La vegetación original de este sitio era la selva baja caducifolia. Actualmente, el suelo tiene diferentes usos, principalmente para asentamientos humanos y, en menor grado, para ganadería y agricultura.

Por su parte, San José Tecoh está dentro de la periferia de la ciudad. Actualmente, la zona se encuentra completamente urbanizada. La vegetación original era selva baja caducifolia, pero ha sido substituida por plantas domesticadas u ornamentales (23).

En ambas localidades pueden observarse abundantes desperdicios orgánicos e inorgánicos, y numerosos perros y gatos sin dueño. Además, algunas calles carecen de servicios básicos como drenaje, luz eléctrica y recolección de basura.

\section{Captura de roedores y toma de muestras biológicas}

Para la captura de los roedores se consideraron 30 manzanas por barrio (60 en total), en las cuales se seleccionó por conveniencia una vivienda. Se colocaron seis trampas Sherman $\left(8 \times 23 \times 9 \mathrm{~cm}\right.$; HB Sherman Traps Inc. ${ }^{\mathrm{TM}}$, Tallahasse, Florida, USA) en cada una de ellas y se mantuvieron activas durante tres noches consecutivas, previa autorización de los habitantes. Los muestreos se alternaron semanalmente por barrio (dos semanas por mes), en el periodo comprendido entre mayo y octubre de 2013.

Las trampas se situaron en lugares con potencial de refugio $o$ alimentación para los roedores y se cebaron con hojuelas de avena y saborizante artificial de vainilla. Se revisaron durante las mañanas y aquellas con captura se reemplazaron por otras colocadas en el mismo lugar. Todas las trampas se retiraron al cuarto día de muestreo.

Los roedores capturados se trasladaron y se procesaron en las instalaciones del Laboratorio de Zoología y del Laboratorio de Parasitología, Unidad de Diagnóstico, Campus de Ciencias Biológicas y Agropecuarias de la Universidad Autónoma de Yucatán. Se determinaron las especies y se recolectaron datos morfométricos, pesos y edades. Los criterios empleados para establecer la edad fueron los propuestos por Hernández-Betancourt, et al. (24).

Los roedores capturados se anestesiaron con pentobarbital sódico (dosis de $60 \mathrm{mg} / \mathrm{kg}$ ) por vía intraperitoneal (25) y se les practicó la eutanasia por dislocación cervical (método que no ocasiona lesiones microscópicas en el corazón), según los lineamientos de la American Veterinary Medical Association (26) y la normatividad nacional vigente de especificaciones 
técnicas para la producción, cuidado y uso de animales de laboratorio (NOM033-ZOO-1995; NOM-062-1999). Durante el transporte y la eutanasia, los animales recibieron un trato humanitario, protegiendo su salud y minimizando el dolor o el estrés generado.

La captura de los animales se llevó a cabo con la autorización de la Secretaría de Agricultura, Ganadería, Desarrollo Rural, Pesca y Alimentación de México (registro de acta: SGPA/DGVS/02528/13). En todos los procedimientos se usó equipo de protección personal para prevenir la exposición a excreciones (es decir, orina, heces y saliva) de los animales. En el sitio de procesamiento, se emplearon guantes dobles de látex, mascarilla desechable, bata y zapatos cerrados.

Después de la eutanasia, se practicó una necropsia con la finalidad de observar lesiones macroscópicas indicativas de la enfermedad de Chagas y para recolectar las secciones de corazón empleadas en el presente estudio. Para ello, la mitad inferior del corazón (ventrículos) se conservó en casetes

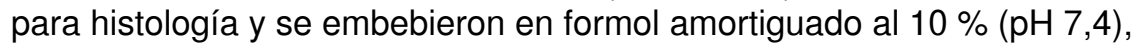
en una proporción de una parte de tejido por diez de fijador, por lo menos, durante 24 horas. No fue posible aprovechar otros tejidos u órganos, ya que se destinaron a objetivos de investigación distintos al del presente estudio. Por otra parte, las aurículas cardiacas se conservaron a $-80^{\circ} \mathrm{C}$ y se utilizaron en el diagnóstico molecular de T. cruzi (16), análisis que no formaba parte de los objetivos de la investigación de la cual se desprendió el presente trabajo.

Las muestras biológicas se etiquetaron con claves que contenían el número consecutivo del individuo capturado, la especie (Mm: M. musculus y Rr: $R$. rattus), el sitio (nombre del barrio) y la fecha de captura.

\section{Estudio histopatológico}

Las secciones de corazón (aurículas) reservadas para el estudio histopatológico se procesaron en el Laboratorio de Patología Acuática del Centro de Investigación y de Estudios Avanzados, Unidad Mérida, y en el Laboratorio de Docencia del Campus de Ciencias Biológicas y Agropecuarias de la Universidad Autónoma de Yucatán.

La técnica que se utilizó para procesarlas fue la descrita por el Armed Forces Institute of Pathology para la inclusión de tejidos y la formación de bloques en parafina (27): se hicieron cortes de $6 \mu \mathrm{m}$ de grosor y se tiñeron con la técnica habitual de hematoxilina y eosina.

Los tejidos se observaron con ayuda de un microscopio óptico convencional usando los objetivos 5X, 10X, 40X y 100X (Eclipse E100, Nikon ${ }^{\mathrm{TM}}$, Tokio, Japón). Las lesiones detectadas se caracterizaron según los criterios de Torres-Castro, et al. $(21,28)$ y Trigo, et al. (29). Cada lesión se describió y se registró de forma individual, pues en ocasiones se presentó más de un tipo de lesión en una sola muestra de tejido cardiaco.

\section{Identificación molecular de Trypanosoma cruzi}

La identificación molecular de T. cruzi se hizo en el Centro de Investigaciones Regionales "Dr. Hideyo Noguchi" (CIR)-UADY. La descripción de la metodología y los resultados en extenso se encuentran en Panti-May, et al. (16). En resumen, con ayuda de un proceso estandarizado, se extrajo ADN total de las aurículas recolectadas, el cual fue utilizado en una reacción en cadena de la polimerasa (PCR) convencional para amplificar un fragmento 
específico de ADN nuclear de T. cruzi con un tamaño de 188 pares de bases (pb). En esta reacción, se emplearon los cebadores desarrollados por Moser, et al. (30): TCZ-1 (3'-CGAGCTCTTGCCCACACGGGTGCT-5') y TCZ-2 (3'-CCTCCAAGCAGCGGATAGTTCAGG -5').

Para validar todas las reacciones, se incluyeron controles negativos (agua estéril) y positivos (ADN de T. cruzi correspondiente al linaje I). Vale la pena mencionar que, debido a la gran sensibilidad y especificidad de los cebadores utilizados en la reacción, no fue necesaria la secuenciación de los fragmentos positivos para confirmar los resultados.

\section{Resultados}

Se capturaron 278 roedores: 165 (59,4\%) M. musculus y $113(40,6 \%) R$. rattus. Del total, $15(5,4 \%)$ muestras de tejido cardiaco resultaron inicialmente positivas para la infección con $T$. cruzi determinada mediante PCR (punto final): ocho $(4,9 \% ; 8 / 165)$ de $M$. musculus y siete $(6,2 \% ; 7 / 113)$ de $R$. rattus.

En la necropsia ninguno de los roedores presentó lesiones macroscópicas concordantes con la enfermedad de Chagas en corazón o en algún otro de los órganos explorados (esófago, intestino grueso o delgado, etc.).

Se detectaron nidos con formas parasitarias (amastigotes) en 7 de las 15 muestras positivas: en 3 de las 8 de $M$. musculus y en 4 de las 7 de $R$. rattus (figura 1). En ambas especies, se encontraron infiltrados inflamatorios, compuestos principalmente por histiocitos y linfocitos, en todas las muestras positivas de miocardio (figura 2). Otras lesiones significativas fueron degeneración de las fibras cardiacas (8/15) (figura 3), congestión de los vasos sanguíneos (6/15) (figura 1) y necrosis celular (5/15) (figura 4).

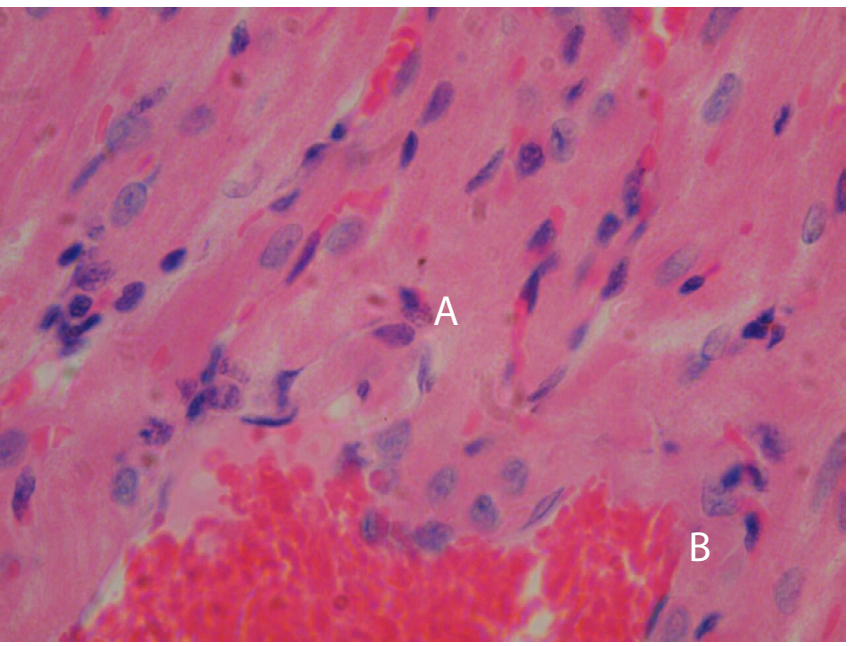

Figura 1. Nido parasitario con amastigotes (A) y congestión de vasos sanguíneos (B) en tejido cardiaco de Rattus rattus naturalmente infectado con Trypanosoma cruzi, capturado en un ambiente suburbano de Mérida, Yucatán, México. Hematoxilina y eosina, 100X

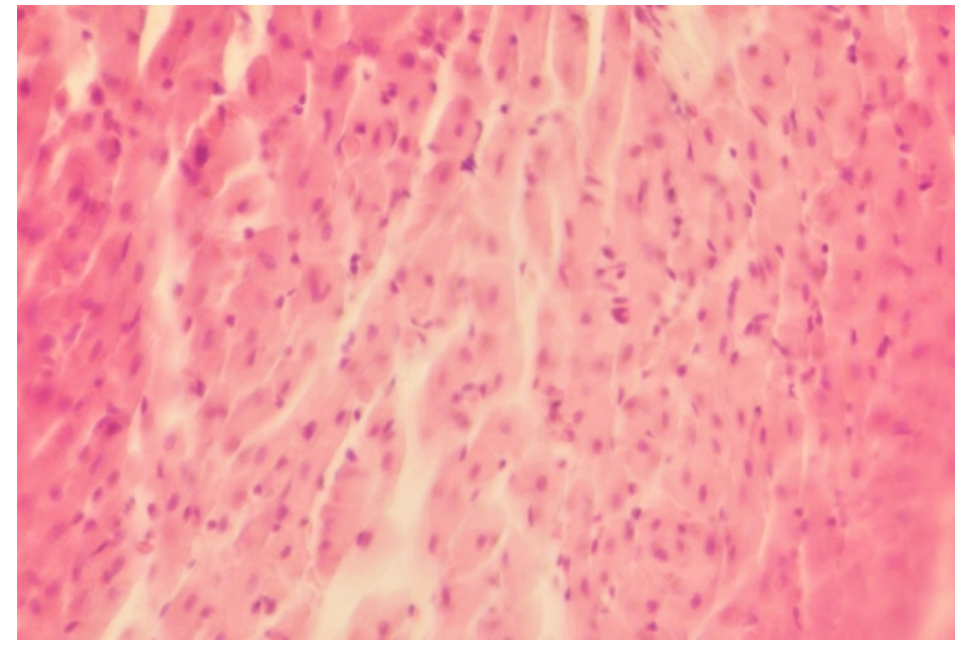

Figura 2. Infiltrado inflamatorio linfocitario multifocal moderado crónico, compuesto por linfocitos e histiocitos en tejido cardiaco de Mus musculus naturalmente infectado con Trypanosoma cruzi, capturado en un ambiente suburbano de Mérida, Yucatán, México. Hematoxilina y eosina, 40X. 


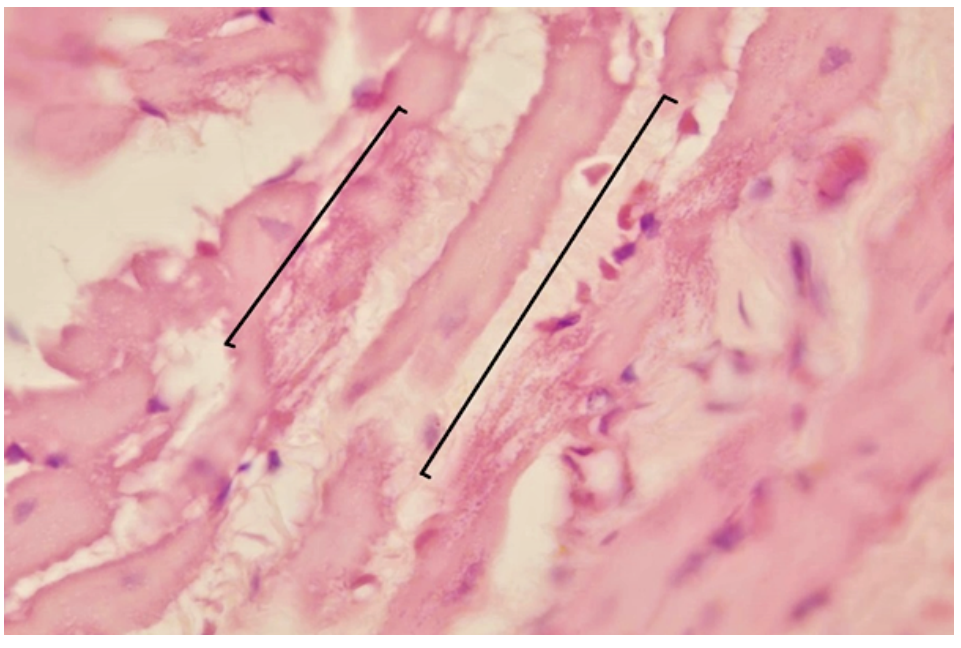

Figura 3. Fibras cardiacas (corchetes) con degeneración celular en tejido cardiaco de Rattus rattus naturalmente infectado con Trypanosoma cruzi, capturado en un ambiente suburbano de Mérida, Yucatán, México. Hematoxilina y eosina, $100 \mathrm{X}$

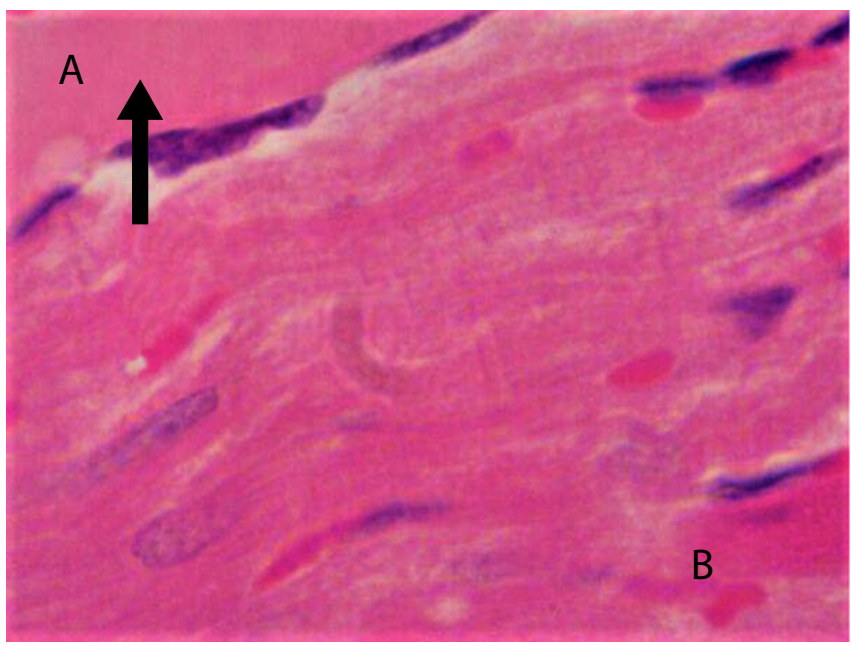

Figura 4. Necrosis por coagulación (A, flecha) en tejido cardiaco de Rattus rattus naturalmente infectado con Trypanosoma cruzi, capturado en un ambiente suburbano de Mérida, Yucatán, México. Nótese la diferencia con la parte de tejido sin daño (B). Hematoxilina y eosina, 100X

Las lesiones microscópicas observadas en los tejidos cardiacos de $M$. musculus se especifican en el cuadro 1 y las de $R$. rattus se presentan en el cuadro 2, los cuales también incluyen la edad y el sexo de los roedores positivos para la infección con $T$. cruzi.

En el cuadro 3, se presentan las frecuencias totales para cada una de las lesiones microscópicas detectadas en los tejidos cardiacos positivos para la infección con $T$. cruzi en los ejemplares de $M$. musculus y $R$. rattus capturados en Mérida.

Cuadro 1. Descripción de las lesiones histológicas observadas en cada una de las muestras de tejido cardiaco positivas para la infección con Trypanosoma cruzi en Mus musculus

\begin{tabular}{|c|c|c|}
\hline Edad & Sexo & Descripción de las lesiones histológicas \\
\hline Adulto & Hembra & $\begin{array}{l}\text { Infiltrado inflamatorio focal moderado crónico conformado por histiocitos, ubicado en el miocardio y asociado a nidos } \\
\text { parasitarios con amastigotes; degeneración celular zonal; congestión vascular }\end{array}$ \\
\hline Adulto & Hembra & $\begin{array}{l}\text { Infiltrado inflamatorio difuso moderado crónico conformado por histiocitos y linfocitos, ubicado en el miocardio, sin } \\
\text { presencia de nidos parasitarios con amastigotes; necrosis focal por coagulación }\end{array}$ \\
\hline Juvenil & Macho & $\begin{array}{l}\text { Infiltrado inflamatorio focal moderado crónico conformado por histiocitos, ubicado en el miocardio y asociado con nidos } \\
\text { parasitarios con amastigotes; degeneración celular multifocal }\end{array}$ \\
\hline Adulto & Macho & $\begin{array}{l}\text { Infiltrado inflamatorio multifocal moderado crónico conformado por histiocitos y linfocitos, ubicado en el miocardio, sin } \\
\text { presencia de nidos parasitarios con amastigotes; degeneración celular multifocal }\end{array}$ \\
\hline Juvenil & Macho & $\begin{array}{l}\text { Infiltrado inflamatorio zonal moderado crónico conformado por histiocitos, ubicado en el miocardio y asociado con nidos } \\
\text { parasitarios con amastigotes; congestión vascular }\end{array}$ \\
\hline Adulto & Macho & $\begin{array}{l}\text { Infiltrado inflamatorio multifocal moderado crónico conformado por histiocitos y linfocitos, ubicado en el miocardio, sin } \\
\text { presencia de nidos parasitarios con amastigotes; necrosis multifocal por coagulación; degeneración celular multifocal }\end{array}$ \\
\hline Juvenil & Macho & $\begin{array}{l}\text { Infiltrado inflamatorio multifocal moderado crónico conformado por histiocitos y linfocitos, ubicado en el miocardio, sin } \\
\text { presencia de nidos parasitarios con amastigotes; degeneración celular multifocal }\end{array}$ \\
\hline Adulto & Hembra & $\begin{array}{l}\text { Infiltrado inflamatorio multifocal moderado crónico conformado por linfocitos, ubicado en el miocardio, sin presencia de } \\
\text { nidos parasitarios con amastigotes; necrosis multifocal por coagulación; degeneración celular zonal; congestión vascular }\end{array}$ \\
\hline
\end{tabular}


Cuadro 2. Descripción de las lesiones histológicas observadas en cada una de las muestras de tejido cardiaco positivas para la infección con Trypanosoma cruzi en Rattus rattus

\begin{tabular}{|c|c|c|}
\hline Edad & Sexo & Descripción de las lesiones histológicas \\
\hline Juvenil & Macho & $\begin{array}{l}\text { Infiltrado inflamatorio multifocal moderado crónico conformado por histiocitos y linfocitos, ubicado en el miocardio y } \\
\text { asociado con nidos parasitarios con amastigotes; congestión vascular }\end{array}$ \\
\hline Juvenil & Macho & $\begin{array}{l}\text { Infiltrado inflamatorio multifocal leve crónico conformado por histiocitos y linfocitos, ubicado en el miocardio y asociado } \\
\text { con nidos parasitarios con amastigotes; degeneración celular multifocal }\end{array}$ \\
\hline Adulto & Macho & $\begin{array}{l}\text { Infiltrado inflamatorio multifocal leve crónico conformado por histiocitos, ubicado en el miocardio, sin presencia de nidos } \\
\text { parasitarios con amastigotes; necrosis multifocal por coagulación; degeneración celular multifocal }\end{array}$ \\
\hline Juvenil & Macho & $\begin{array}{l}\text { Infiltrado inflamatorio multifocal leve crónico conformado por histiocitos, ubicado en el miocardio y asociado con nidos } \\
\text { parasitarios con amastigotes }\end{array}$ \\
\hline
\end{tabular}

Cuadro 3. D. Frecuencia total y por especie de roedor de cada una de las lesiones histológicas observadas en las muestras de tejido cardiaco positivas para la infección con Trypanosoma cruzi

\begin{tabular}{lccc}
\hline & \multicolumn{3}{c}{ Por especie } \\
\cline { 2 - 4 } Lesión histológica & $\mathbf{n}(\%)$ & $\begin{array}{c}\text { Mus musculus } \\
\mathbf{n}(\%)\end{array}$ & $\begin{array}{c}\text { Rattus rattus } \\
\mathbf{n}(\%)\end{array}$ \\
\hline Nidos parasitarios con amastigotes & $8 / 15(53,3)$ & $3 / 8(37,5)$ & $4 / 7(57,1)$ \\
Infiltrado inflamatorio focal moderado crónico & $2 / 15(13,3)$ & $2 / 8(25,0)$ & $0 / 7(0,0)$ \\
Infiltrado inflamatorio difuso moderado crónico & $1 / 15(6,6)$ & $1 / 8(12,5)$ & $0 / 7(0,0)$ \\
Infiltrado inflamatorio multifocal moderado crónico & $7 / 15(46,6)$ & $4 / 8(50,0)$ & $3 / 7(42,8)$ \\
Infiltrado inflamatorio zonal moderado crónico & $1 / 15(6,6)$ & $1 / 8(12,5)$ & $0 / 7(0,0)$ \\
Infiltrado inflamatorio multifocal leve crónico & $4 / 15(26,6)$ & $0 / 8(0,0)$ & $4 / 7(57,1)$ \\
Degeneración celular zonal & $2 / 15(13,3)$ & $2 / 8(25,0)$ & $0 / 7(0,0)$ \\
Degeneración celular multifocal & $7 / 15(46,6)$ & $4 / 8(50,0)$ & $3 / 7(42,8)$ \\
Congestión vascular & $5 / 15(33,3)$ & $3 / 8(37,5)$ & $2 / 7(28,5)$ \\
Necrosis coagulativa focal & $2 / 15(13,3)$ & $1 / 8(12,5)$ & $1 / 7(14,2)$ \\
Necrosis coagulativa multifocal & $3 / 15(20,0)$ & $2 / 8(25,0)$ & $1 / 7(14,2)$ \\
\hline
\end{tabular}

\section{Discusión}

El objetivo del presente trabajo fue describir y reportar los tipos y la frecuencia de las distintas lesiones microscópicas encontradas en el tejido cardiaco de roedores sinantrópicos naturalmente infectados con $T$. cruzi capturados en los barrios suburbanos de Mérida, Yucatán, México. Los estudios histopatológicos en roedores $u$ otros animales en condiciones de laboratorio (infectados experimentalmente) son numerosos y variados (31-37), pero no sucede lo mismo con los realizados en reservorios o huéspedes naturalmente infectados $(20,21,38)$.

En diversos estudios se ha concluido que T. cruzi es una especie heterogénea y que tanto el parasitismo intracelular como el desarrollo de las numerosas lesiones en el miocardio y en otros órganos colonizados, dependen en gran medida de la presencia y el número de nidos parasitarios con amastigotes $(8,39,40)$, los cuales también son indicativos de que, después de instalarse y reconocer los diferentes tejidos blanco, T. cruzi se reproduce intensamente a nivel intracelular dando como resultado un mayor número de parásitos en el torrente sanguíneo después de diferenciarse en tripomastigotes (33). Estos nidos parasitarios se observaron en los 
tejidos cardiacos de los ejemplares de $M$. musculus y $R$. rattus estudiados (figura 1), lo cual concuerda con reportes previos (21) para ambos roedores sinantrópicos capturados en Molas, Yucatán, México, población con características climatológicas y sociales similares a los sitios de estudio considerados en el actual trabajo.

En este estudio los nidos con formas de amastigotes que contenían el clásico complejo núcleo-cinetoplasto, se encontraron en 8 de las 15 muestras de tejido cardiaco positivas en la PCR convencional para la infección con $T$. cruzi (figura 1).

Este menor número podría explicarse con base en los hallazgos de Pizzi, et al. (39), quienes sugieren que la distribución de los nidos parasitarios es mayor en el miocardio de las aurículas, secciones del corazón con las que, infortunadamente, no se contó en este caso. Asimismo, Araujo-Carreira, et al. (38) describieron la presencia de nidos parasitarios en cuatro de diez corazones de zarigüeyas (Didelphis marsupiales) naturalmente infectadas, lo que indicaría que la localización de los nidos no es homogénea en los animales reservorios. En el estudio de Acosta-Viana, et al. (20), los autores tampoco encontraron los nidos en un perro positivo por PCR para la infección con T. cruzi.

Por otro lado, Rossi, et al. (40), señalan que la observación de los nidos con formas de amastigotes se dificulta, incluso en la etapa crónica o indefinida de la enfermedad, y que su ubicación depende en gran medida de los signos o síntomas clínicos. No obstante, en estudios experimentales como el de Castro-Sesquen, et al. (36), los nidos con formas de amastigotes se presentaron a los 25 días de la inoculación de los parásitos adultos en el tejido cardiaco de 18 cuyos (Cavia porcellus) infectados, específicamente en aquellos animales que se encontraban en la etapa crónica de la enfermedad.

Otro aspecto que pudo influir en el porcentaje de detección de nidos con formas de amastigotes en los roedores positivos por PCR, se refiere al hecho de que en el presente estudio no se empleó músculo esquelético, tejido que puede verse ampliamente afectado y ocupado por $T$. cruzi, sobre todo en infecciones crónicas, lo que restringió su distribución a otro órganos o tejidos $(36,41)$. Asimismo, en modelos de ratón se ha observado que es posible que T. cruzi no persista en el tejido cardiaco previamente afectado $(34,42)$, lo cual reduce la probabilidad de detectarlo en la observación histopatológica.

Otro hallazgo del análisis microscópico del presente estudio fue que los nidos parasitarios se presentaron tanto en roedores machos como en hembras, lo cual concuerda con hallazgos previos (21). En este sentido, León, et al. (37), tampoco encontraron diferencias en la distribución de los nidos según el sexo de los animales empleados en tres modelos distintos de ratón.

Las lesiones más frecuentes (15/15) en el tejido cardiaco de los roedores positivos para la infección con $T$. cruzi fueron los infiltrados inflamatorios, conformados principalmente por linfocitos e histiocitos (figura 2), aunque con diferente grado de gravedad y distribución. Esta lesión se ha descrito en el $100 \%$ de los corazones de los pacientes humanos con enfermedad de Chagas $(41,43)$ y se relaciona con otros daños cardiacos de las fases crónica o indeterminada de la enfermedad $(44,45)$, por lo que es probable que los animales utilizados en este trabajo se encontraran en dichas etapas y pudieran estar predispuestos a la infección, o no estarlo (37); sin embargo, esta hipótesis debe demostrarse con base en estudios experimentales posteriores. Asimismo, los infiltrados inflamatorios se han descrito en 
estudios en modelos de ratón directamente vinculados con la carga parasitaria y no con el genotipo o linaje del parásito infeccioso $(32,37,39,46)$, lo que tampoco pudo determinarse en el presente estudio.

Por otra parte, este tipo de lesiones se han asociado con el desarrollo de necrosis (figura 4) de las fibras cardiacas afectadas y con la mortalidad subsiguiente $(40,43)$.

Se han determinado diversos factores como detonantes de la formación y presentación de infiltrados inflamatorios celulares en los individuos con infecciones crónicas, entre los que pueden mencionarse la patogenia del genotipo de $T$. cruzi infeccioso, el tiempo de evolución de la enfermedad y la presencia de infección concomitante con otros parásitos (poliparasitismo) (47), así como la interacción del genoma del parásito con el del huésped infectado, la edad, el sexo y la presencia de infecciones previas (48).

Otras lesiones detectadas en el presente estudio fueron necrosis por coagulación (figura 4), degeneración celular (figura 3) y congestión vascular (figura 1), todas las cuales fueron también reportadas por Torres-Castro, et al. (21) en $M$. musculus y $R$. rattus, y han sido identificadas en modelos de ratón $(49,50)$, en zarigüeyas de cola corta (Monodelphis domestica) (51), y en humanos con enfermedad crónica y con compromiso del sistema circulatorio $(40,52)$.

Aunque algunas de las lesiones encontradas en el presente estudio histopatológico pueden ser ocasionadas por otros agentes infecciosos circulantes en los roedores empleados (por haber sido capturados en un ambiente periurbano) (28), los resultados de Panti-May, et al. (16) y los de este estudio permiten concluir que los roedores $M$. musculus y $R$. rattus son reservorios de $T$. cruzi y que, probablemente, están involucrados en el ciclo de infección de la tripanosomiasis americana en la región. Se recomienda adelantar estudios que permitan conocer cuáles son los distintos linajes de T. cruzi circulantes en las poblaciones de roedores y otros reservorios animales de Yucatán, México.

Después de revisar la literatura consultada, puede señalarse que este es el primer estudio sistemático que reporta y describe los distintos tipos de lesiones histopatológicas del tejido cardiaco de roedores sinantrópicos naturalmente infectados con T. cruzi capturados en Yucatán, México.

\section{Agradecimientos}

A los biólogos Josué Meza Sulú y Lorenzo Sodá Tamayo, por su invaluable ayuda en el trabajo de campo, así como a todas las familias participantes que nos dieron el permiso de entrar a sus hogares.

\section{Referencias}

1. López-Cancino SA, Tun-Ku E, de la Cruz-Feliz HK, Ibarra-Cerdeña CN, Izeta-Alberdi A, Pech-May A, et al. Landscape ecology of Trypanosoma cruzi in the southern Yucatán Peninsula. Acta Trop. 2015;151:58-72. https://doi.org/10.1016/j.actatropica.2015.07.021

2. Ramsey JM, Townsend-Peterson A, Carmona-Castro O, Moo-Llanes DA, Nakazawa Y, Butrick M, et al. Atlas of Mexican Triatominae (Reduviidae: Hemiptera) and vector transmission of Chagas disease. Mem Inst Oswaldo Cruz. 2015;110:339-52. https://doi.org/10.1590/0074-02760140404

3. Moncayo A, Ortiz-Yanine MI. An update on Chagas disease (human American trypanosomiasis). Ann Trop Med Parasitol. 2006;100:663-77.

4. Leite MF, Moyer MS, Andrews NW. Expression of the mammalian calcium signaling response to Trypanosoma cruzi in Xenopus laevis oocytes. Mol Biochem Parasitol. 1998;92:1-13. https://doi.org/10.1016/S0166-6851(97)00211-9 
5. Prata A. Clinical and epidemiological aspects of Chagas disease. Lancet Infect Dis. 2001;1:92-100. https://doi.org/10.1016/S1473-3099(01)00065-2

6. Carrada-Bravo T. Trypanosoma cruzi: historia natural y diagnóstico de la enfermedad de Chagas. Rev Mex Patol Clin. 2004;51:205-19.

7. Bonney KM, Engman DM. Chagas heart disease pathogenesis: One mechanism or many? Curr Mol Med. 2008;6:510-8. https://doi.org/10.2174/156652408785748004

8. Postan M, Dvorak JA, McDaniel JP. Studies of Trypanosoma cruzi clones in bred mice. I. A comparison of the course of infection of $\mathrm{C} 3 \mathrm{H} / \mathrm{HeN}$ mice with two clones isolated from a common source. Am J Trop Med Hyg. 1983;32:497-506. https://doi.org/10.4269/ajtmh.1984.33.236

9. Andrade LO, Galvão LM, Meirelles MN, Chiari E, Pena SD, Macedo AM. Differential tissue tropism of Trypanosoma cruzi strains: An in vitro study. Mem Inst Oswaldo Cruz. 2010;105:834-7. https://doi.org/10.1590/S0074-02762010000600018

10. Rozas M, Botto-Mahan C, Coronado X, Ortiz S, Cattan PE, Solari A. Coexistence of Trypanosoma cruzi genotypes in wild and peridomestic mammals in Chile. Am J Trop Med Hyg. 2007;77:643-53.

11. Rademaker V, Herrera HM, Raffel TR, D'Andrea PS, Freitas TPT, Abreu UG, et al. What is the role of small rodents in the transmission cycle of Trypanosoma cruzi and Trypanosoma evansi (Kinetoplastida Trypanosomatidae)? A study case in the Brazilian Pantanal. Acta Trop. 2009;111:102-7. https://doi.org/10.1016/j.actatropica.2009.02.006

12. Brigada AM, Doña R, Caviedes-Vidal E, Moretti E, Basso B. American Tripanosomiasis: A study on the prevalence of Trypanosoma cruzi and Trypanosoma cruzi-like organisms in wild rodents in San Luis province, Argentina. Rev Soc Bras Med Trop. 2010;43:249-53 https://doi.org/10.1590/S0037-86822010000300007

13. Alemán A, Guerra T, Maikis TJ, Milholland MT, Castro-Arellano I, Forstner MR, et al. The prevalence of Trypanosoma cruzi, the causal agent of Chagas disease, in Texas rodent populations. Ecohealth. 2017;14:130-143. https://doi.org/10.1007/s10393-017-1205-5

14. Gürtler RE, Cardinal MV. Reservoir host competence and the role of domestic and commensal hosts in the transmission of Trypanosoma cruzi. Acta Trop. 2015;151:32-50. https://doi.org/10.1016/j.actatropica.2015.05.029

15. Zavala-Velázquez J, Barrera-Pérez M, Rodríguez-Félix ME, Guzmán-Marín E, Ruíz-Piña $\mathrm{H}$. Infection by Trypanosoma cruzi in mammals in Yucatán, México: A serological and parasitological study. Rev Inst Med Trop Sao Paulo. 1996;38:289-92.

16. Panti-May JA, de Andrade RR, Gurubel-González Y, Palomo-Arjona E, Sodá-Tamayo L, Meza-Sulú J, et al. A survey of zoonotic pathogens carried by house mouse and black rat populations in Yucatán, México. Epidemiol Infect. 2017;145:2287-95. https://doi.org/10.1017/S0950268817001352

17. Panti-May JA, Hernández-Betancourt SF, Torres-Castro MA, Machaín-Williams C, CigarroaToledo N, Sodá L, et al. Population characteristics of human-commensal rodents present in households from Mérida, Yucatán, México. MANTER: Journal of Parasite Biodiversity. 2016;5. https://doi.org/10.13014/K2VD6WCX

18. Organización Panamericana de la Salud (OPS). Guía para vigilancia, prevención, control y manejo clínico de la Enfermedad de Chagas aguda transmitida por alimentos. Fecha de consulta: 10 de octubre de 2017. Disponible en: http://bvs.panalimentos.org/local/File/ Guia_Enfermedad_Chagas_2009esp.pdf

19. de Diego JA, Palau MT, Gamallo C, Penin P. Relationships between histopathological findings and phylogenetic divergence in Trypanosoma cruzi. Trop Med Int Health. 1998;3:222-33. https://doi.org/10.1111//.1365-3156.1998.tb00275.x

20. Acosta-Viana KY, Guzmán-Marín E, Jiménez-Coello M, Torres-León MA, Colín-Flores RF, Ortega-Pacheco A. Cardiac lesions in naturally infected dogs with Trypanosoma cruzi. J Agr Sci Tech. 2011;A1:932-8.

21. Torres-Castro MA, Hernández-Betancourt SF, Torres-León MA, Puerto FI. Lesiones histológicas asociadas a la posible infección por Trypanosoma cruzi (Chagas, 1909) en corazones de roedores sinantrópicos capturados en Yucatán, México. An Biol. 2016;38:29-35.

22. Orellana R, Islebe G, Espadas C. Presente, pasado y futuro de los climas de la Península de Yucatán. Naturaleza y sociedad en el área Maya. Pasado, presente y futuro. Primera edición. México, DF: Academia Mexicana de Ciencias, CICY; 2003. p. 37-52. 
23. Flores JS, Espejel I. Tipos de vegetación de la Península de Yucatán. Etnoflora Yucatanense, Fascículo 3. Primera edición. Mérida, México: Universidad Autónoma de Yucatán; 1994. p. 135.

24. Hernández-Betancourt S, Cimé-Pool J, Medina-Peralta S, González-Villanueva M. Fluctuación poblacional de Ototylomys Phyllotis Merriam, 1901 (Rodentia: Muridae) en una selva mediana subcaducifolia del sur de Yucatán, México. Acta Zool Mex. 2008;24:161-77.

25. Gaertner DJ, Hallman TM, Hankenson FC, Batchelder MA. Anesthesia and analgesia for laboratory rodents. En: Fish RE, Brown MJ, Danneman PJ, Karas AZ, editors. Anesthesia and analgesia in laboratory animals. Second edition. London: Elsevier; 2008. p. 239-97.

26. Leary S, Underwood W, Anthony R, Cartner S, Corey D, Grandin T, et al. AVMA Guidelines for the Euthanasia of Animals: 2013 edition. Schaumburg, IL: American Veterinary Medical Association; 2013. p.102.

27. Armed Forces Institute of Pathology (AFIP). Methods in Histotechnology. Third edition. Washington, D.C.: American Registry of Pathology; 1992. p.279.

28. Torres-Castro M, Guillermo-Cordero L, Hernández-Betancourt S, Gutiérrez-Ruíz E, AgudeloFlórez P, Peláez-Sánchez R, et al. First histopathological study in kidneys of rodents naturally infected with Leptospira pathogenic species from Yucatán, México. Asian Pac J Trop Med. 2016;9:145-7. https://doi.org/10.1016/j.apjtm.2016.01.018

29. Trigo T, Valero E. Patología general veterinaria. Cuarta edición. México, D.F.: Servicios editoriales de la Universidad Autónoma de México; 2004. p. 437.

30. Moser DR, Kirchhoff LV, Donelson JE. Detection of Trypanosoma cruzi by DNA amplification using the polymerase chain reaction. J Clin Microbiol. 1989;27:1477-82.

31. Andrade ZA, Andrade SG, Correa R, Sadigursky M, Ferrans VJ. Myocardial changes in acute Trypanosoma cruzi infection. Ultrastructural evidence of immune damage and the role of microangiopathy. Am J Pathol. 1994;144:1403-11.

32. Zúñiga $\mathrm{C}$, Vargas R, Vergara U. Evolución de la infección con Trypanosoma cruzi en cepas susceptibles y resistentes de ratones. Arch Med Vet. 2002;34:183-8. https://doi.org/10.4067/S0301-732X2002000200004

33. Díaz-Limay E, Escalante H, Jara CA. Niveles de parasitemia y alteraciones histopatológicas en Mus musculus BALB/c infectado con Trypanosoma cruzi obtenido de Panstrongylus chinai del Valle Chamán, La Libertad - Perú. Parasitol Latinoam. 2004;59:153-8. https://doi.org/10.4067/S0717-77122004000300011

34. Marinho CR, Bucci DZ, Dagli ML, Bastos KR, Grisotto MG, Sardinha LR, et al. Pathology affects different organs in two mouse strains chronically infected by a Trypanosoma cruzi clone: A model for genetic studies of Chagas' disease. Infect Immun. 2004;72:2350-7. https://doi.org/10.1128/IAl.72.4.2350-2357.2004

35. Roellig DM, Yabsley MJ. Infectivity, pathogenicity, and virulence of Trypanosoma cruzi isolates from sylvatic animals and vectors, and domestic dogs from the United States in ICR strain mice and SD strain rats. Am J Trop Med Hyg. 2010;83:519-22. https://doi.org/10.4269/ajtmh.2010.09-0663

36. Castro-Sesquen YE, Gilman RH, Yauri V, Angulo N, Verastegui M, Velásquez DE, et al. Cavia porcellus as a model for experimental infection by Trypanosoma cruzi. Am J Pathol. 2011;179:281-8. https://doi.org/10.1016/j.ajpath.2011.03.043

37. León CM, Montilla M, Vanegas R, Castillo M, Parra E, Ramírez JD. Murine models susceptibility to distinct Trypanosoma cruzi I genotypes infection. Parasitology. 2017;144:512-9. https://doi.org/10.1017/S0031182016001980

38. Araujo-Carreira JC, Jansen AM, Deane MP, Lenzi HL. Histopathological study of experimental and natural infections by Trypanosoma cruzi in Didelphis marsupialis. Mem Inst Oswaldo Cruz. 1996;91:609-18. https://doi.org/10.1590/S0074-02761996000500012

39. Pizzi T, Wallace RA, Villagra OR, Muñoz VS, Ortiz ZS, Solari IA. Concordancia de lesiones histológicas en ratones infectados por dos poblaciones de Trypanosoma cruzi de Chile. Rev Med Chile. 2005;133:432-8. https://doi.org/10.4067/S0034-98872005000400006

40. Rossi MA, Tanowitz HB, Malvestio LM, Celes MR, Campos EC, Blefari V, et al. Coronary microvascular disease in chronic Chagas cardiomyopathy including an overview on history, pathology, and other proposed pathogenic mechanism. PLoS Negl Trop Dis. 2010;4:e674. https://doi.org/10.1371/journal.pntd.0000674 
41. Bazan C, Micucci L, Romina F, Triquel MF, Lo Presti MS, Baez A, et al. Persistence of Trypanosoma cruzi in experimental chagasic cardiomyopathy. Anti-Infective Agents. 2012;10:136-41. https://doi.org/10.2174/2211362611208020136

42. Andrade LO, Machado CR, Chiari E, Pena SD, Macedo AM. Differential tissue distribution of diverse clones of Trypanosoma cruzi in infected mice. Mol Biochem Parasitol. 1999;100:163-72. https://doi.org/10.1016/S0166-6851(99)90035-X

43. Moreno-Medina E, Valerio-Campos I, Goyenaga-Castro P. Miocarditis y miocardiopatía dilatada por Trypanosoma cruzi: reporte de un caso. Parasitol Latinoam. 2007;62:148-53. https://doi.org/10.4067/S0717-77122007000200008

44. Zhang L, Tarleton RL. Parasite persistence correlates with disease severity and localization in chronic Chagas' disease. J Infect Dis. 1999;180:480-6. https://doi.org/10.1086/314889

45. Kierszenbaum F. Mechanisms of pathogenesis in Chagas disease. Acta Parasitol. 2007;52:1-12. https://doi.org/10.2478/s11686-006-0048-y

46. Cruz L, Vivas A, Montilla M, Hernández C, Flórez C, Parra E, et al. Comparative study of the biological properties of Trypanosoma cruzi I genotypes in a murine experimental model. Infect Genet Evol. 2015;29:110-117. https://doi.org/10.1016/j.meegid.2014.11.012

47. Jansen AM, Carreira JC, Deane MP. Infection of a mammal by monogenetic insect trypanosomatids (kynetoplastida, trypanosomatidae). Mem Inst Oswaldo Cruz. 1988;3:2712. https://doi.org/10.1590/S0074-02761988000300001

48. Tarleton R. Chagas disease: A role for autoimmunity? Trends Parasitol. 2003;19:447-51. https://doi.org/10.1016/j.pt.2003.08.008

49. Villela-Ribeiro LC, Barbosa AA Jr, Andrade ZA. Pathology of intracardiac nerves in experimental Chagas disease. Mem Inst Oswaldo Cruz. 2002;97:1019-25. https://doi.org/10.1590/S0074-02762002000700016

50. Rendón DA, Genes CM, Triana O. Lesión celular del miocardio y actividad de la ATPsintasa mitocondrial en ratas infectadas con una cepa colombiana de Trypanosoma cruzi. Biomédica. 2007;27(Sup.1):40-9. https://doi.org/10.7705/biomedica.v27i1.247

51. Roellig DM, McMillan K, Ellis AE, Vandeberg JL, Champagne DE, Yabsley MJ. Experimental infection of two South American reservoirs with four distinct strains of Trypanosoma cruzi. Parasitology. 2010;137:959-66. https://doi.org/10.1017/S0031182009991995

52. Teixeira AR, Nascimiento RJ, Sturm NR. Evolution and pathology in Chagas disease-A review. Mem Inst Oswaldo Cruz. 2006;101:463-91. http://dx.doi.org/10.1590/S0074-02762006000500001 